\title{
DINÂMICA COMPLEXA NO SISTEMA BROMATO/HIPOFOSFITO/ACETONA/MANGANÊS E FERROÍNA
}

\author{
Gustavo Tokoro, Hyrla C. L. Oliveira e Hamilton Varela* \\ Instituto de Química de São Carlos, Universidade de São Paulo, CP 780, 13560-970 \\ São Carlos-SP, Brasil
}

Recebido em 19/12/06; aceito em 10/4/07; publicado na web em 25/10/07

\begin{abstract}
COMPLEX DYNAMICS IN THE BROMATE/HYPOPHOSPHITE/ACETONE/MANGANESE AND FERROIN SYSTEM. New chemical systems have been recently designed for the study of complex phenomena such as oscillatory dynamics in the temporal domain and spatiotemporal pattern formation. Systems derived from oscillators based on the chemistry of bromate are the most extensively studied, with the celebrated Belousov-Zhabotinsky (BZ) reaction being the most popular example. Problems such as the formation of bubbles $\left(\mathrm{CO}_{2}\right)$ and solid precipitate in the course of the reaction and the occurrence of simply short-lived oscillations under batch conditions are very common and, in some cases, compromise the use of some of these systems. It is investigated in this paper the dynamic behavior of the bromate/hypophosphite/acetone/dual catalyst system, which has been sugested as an interesting alternative to circumvent those inconvenients. In this work, manganese and ferroin are employed as catalysts and the complete system $\left(\mathrm{BrO}_{3}^{-} / \mathrm{H}_{2} \mathrm{PO}_{2}^{-} /\right.$acetone/Mn(II)-ferroin) is studied under batch conditions. Temporal symmetry breaking was studied in a reactor under agitation by means of simultaneous records of the potential changes of platinum and $\mathrm{Ag} / \mathrm{AgBr}$ electrodes, both measured versus a reversible hydrogen electrode. Additionally, spatio-temporal formation of target patterns and spiral waves were obtained when the oscillating mixture was placed in a quasi two-dimensional reactor.
\end{abstract}

Keywords: oscillations; chemical kinetics; pattern formation.

\section{INTRODUÇÃO}

\section{Propriedades emergentes e sistemas complexos}

Define-se formalmente comportamento emergente como "comportamento coletivo de um agregado de subunidades fisicamente similares não observado individualmente por nenhuma sub-unida$\mathrm{de}{ }^{1}$. Em um contexto mais amplo, Korn ${ }^{2}$ compilou as principais definições de propriedades emergentes como sendo dadas em termos: da não aditividade das suas partes; de uma novidade, algo simplesmente novo; da não dedutibilidade e, da não preditabilidade. Finalmente, o autor ${ }^{2}$ encerra que emergência é a nova associação que conduz a novas e estáveis configurações observáveis. Emergência é a propriedade mais importante dos sistemas complexos. O comportamento de um sistema complexo não pode ser entendido em termos apenas da extrapolação das propriedades dos seus componentes individuais, ou seja, as propriedades que surgem no nível macroscópico não podem ser preditas a partir das propriedades dos componentes microscópicos, como apresentado no artigo clássico More is different ${ }^{3}$.

Whitesides e Ismagilov ${ }^{4}$ classificaram um sistema complexo como um sistema no qual: sua evolução é muito sensível às condições iniciais ou a pequenas perturbações; o número de componentes independentes interagindo é grande ou, um no qual há vários caminhos através dos quais a evolução do sistema pode proceder. Comportamento complexo expresso na forma de cinética oscilatória e estruturação espacial, por exemplo, é comum em muitos sistemas reacionais mantidos suficientemente afastados do estado de equilíbrio termodinâmico.

O estudo da emergência, seleção e evolução de padrões espaço-temporais em sistemas físico-químicos complexos tem atraído

*e-mail: varela@iqsc.usp.br bastante interesse recentemente. Uma grande motivação para tais estudos reside na utilização de análogos químicos como modelos funcionais ${ }^{5}$ no estudo de padrões de atividade em sistemas biológicos, obviamente, bem mais complexos e menos tratáveis ${ }^{6}$. Exemplos de sistemas físico-químicos, cujas propriedades emergentes vêm sendo estudadas recentemente incluem processos heterogêneos nas interfaces sólido/gás ${ }^{7-9}$ e sólido/líquido ${ }^{10-12}$, assim como reações homogêneas como no caso dos osciladores da família do bromato $^{13-16}$.

Em sistemas homogêneos, fenômenos temporais complexos na forma de oscilações ou multi-estabilidade são observados em reatores volumétricos com a solução mantida sob agitação, enquanto auto-organização espaço-temporal como ondas químicas e frentes reacionais se manifestam em sistemas estagnados, em condições nas quais termos reacionais cooperam com termos de transporte. Em geral, as seguintes características são desejáveis para um sistema químico homogêneo ser utilizado na investigação da dinâmica de padrões espaço-temporais bidimensionais em uma camada fina de solução ${ }^{17}$ : oscilar em batelada a temperatura ambiente por um período considerável; não produzir produtos gasosos ou sólidos (precipitados); apresentar formação de ondas químicas e padrões espaço-temporais facilmente visíveis e com comprimento de onda e velocidade adequados; ser susceptível à perturbação ou ao controle, por exemplo, fotoquimicamente e, apresentar um mecanismo homogêneo relativamente simples que permita modelagem e simulação numérica.

\section{A Reação de Belousov-Zhabotinsky}

A reação de Belouzov-Zhabotinsky (BZ) tem desempenhado um papel fundamental nas investigações sobre dinâmica complexa em reações químicas ${ }^{13-15}$, sendo o protótipo mais famoso da família dos osciladores envolvendo bromato ${ }^{16}$. Essa reação en- 
volve a oxidação de uma espécie orgânica (normalmente ácido malônico, AM) por bromato, catalisada por um íon metálico como $\mathrm{Ce}(\mathrm{III}), \mathrm{Mn}(\mathrm{II})$ ou ferroína (Fe(phen) ${ }_{3}{ }^{2+}$ ), em meio contendo ácido sulfúrico. A grande energia livre de Gibbs associada à oxidação de ácido malônico por bromato acidificado é a força motriz da reação. Em termos cinéticos, entretanto, essa reação é bastante lenta e precisa ser catalisada pelo íon metálico. O mecanismo proposto originalmente por Field, Korös e Noyes (FKN) em $1972^{18}$ tem sido bastante utilizado como modelo básico da reação de BZ. Nesse mecanismo, bromato e ácido malônico são consumidos e ácido bromomalônico e $\mathrm{CO}_{2}$ são produzidos. Oscilações resultam das variações periódicas nas concentrações do ativador, $\mathrm{HBrO}_{2}$, que catalisa sua própria produção (gerando, portanto, um ciclo de retro-alimentação positiva), e do inibidor, $\mathrm{Br}^{-}$, que regula o processo autocatalítico (retro-alimentação negativa). A geração de $\mathrm{CO}_{2}$ em geral ocorre como um subproduto das reações do ácido bromomalônico.

Juntamente com a produção de precipitados, a geração de bolhas no decorrer da reação é considerada um problema crítico no estudo do comportamento dinâmico em osciladores químicos. De fato, o desprendimento de produto gasoso perturba não apenas a observação de estruturas espaço-temporais em sistemas estagnados, mas também a investigação de transições temporais sutis em sistemas mantidos sob agitação. Esforços têm sido realizados na tentativa de mitigar a formação de bolhas em sistemas químicos com base nos osciladores envolvendo bromato ${ }^{17,19,20}$. A substituição do ácido malônico por outro substrato orgânico tem sido apontada como uma alternativa eficiente na supressão da formação de bolhas ${ }^{17,21-23}$. Diferentemente dos sistemas contendo acetilacetona ${ }^{21} \mathrm{e}$ etilacetoacetato ${ }^{22}$, por exemplo, além de suprimir o desprendimento de bolhas não há formação de precipitados quando acetona é utilizada como substrato orgânico.

Quando utilizada como catalisador, a ferroína é oxidada ao complexo de ferro III, ferrína, resultando em uma mudança de coloração de vermelho para azul. Algumas vantagens importantes do sistema catalisado pela ferroína em comparação à receita original com cério $\mathrm{são}^{24}$ : a presença de oscilações de grande amplitude no potencial redox de um eletrodo de platina, em fase com as mudanças de coloração e, as oscilações são geralmente observadas em uma ampla faixa de concentrações. Não obstante algumas similaridades, está claro que em variantes da reação de BZ utilizando ferroína como catalisador diferentes aspectos mecanísticos adicionais ao sistema tradicional com cério devem ser levadas em conta, de forma que a dinâmica não é capturada simplesmente pelo mecanismo $\mathrm{FKN}^{18}$.

Em um contexto mais amplo, sistemas espacialmente estendidos utilizando ferroína podem ainda ser empregados como modelo aos estudos sobre o impacto do acoplamento global na emergência, seleção e evolução de estruturas dissipativas. Especificamente, o acoplamento global pode ser modulado graças à fotossensibilidade da ferriína, já que a luz visível $(550-660 \mathrm{~nm})$ catalisa a redução de ferriína para ferroína:

$\mathrm{Fe}(\text { phen })_{3}{ }^{3+}+\mathrm{h} v \rightarrow \mathrm{Fe}(\text { phen })_{3}{ }^{2+}$

de forma que as oscilações podem ser suprimidas ou induzidas por iluminação ${ }^{14}$. Pode-se afirmar o efeito de iluminação tem sido pouquíssimo explorado nesse sistema até então ${ }^{25,26}$. Tais investigações assumem importância vital tendo em vista que estudos sobre a formação e manipulação de padrões espaço-temporais na presença de diferentes tipos de acoplamentos espaciais é considerada atualmente uma das áreas mais ativas de pesquisa no ramo de auto-organização dinâmica ${ }^{11,12,27}$.

\section{$\mathrm{O}$ sistema: $\mathrm{BrO}_{3}^{-} / \mathrm{H}_{2} \mathrm{PO}_{2}^{-} /$acetona/dois catalisadores}

Tomando como base o oscilador $\mathrm{BrO}_{3}^{-} / \mathrm{H}_{2} \mathrm{PO}_{2}^{-} / \mathrm{Mn}(\mathrm{II}) / \mathrm{N}_{2}$, Orban e colaboladores ${ }^{17}$ sugeriram recentemente o sistema $\mathrm{BrO}_{3}^{-} / \mathrm{H}_{2} \mathrm{PO}_{2}^{-} /$ acetona/dois catalisadores como uma alternativa bastante interessante às receitas envolvendo variantes da reação de BZ, particularmente em termos das oscilações sustentadas por longo tempo em regime de batelada, ausência de produtos gasosos ou sólidos e a formação de padrões espaço-temporais facilmente visíveis. Nesse sistema, Mn(II) é utilizado como um dos catalisadores e $\mathrm{Ru}(\mathrm{bpy})_{3} \mathrm{SO}_{4}$, ferroína ou difenilamina serve como catalisador secundário.

São apresentados nesse trabalho resultados da dinâmica espaço-temporal do sistema $\mathrm{BrO}_{3}^{-} / \mathrm{H}_{2} \mathrm{PO}_{2}^{-} /$acetona/Mn(II)-ferroína operado em regime de batelada. $\mathrm{O}$ sistema foi estudado em domínio temporal utilizando um reator volumétrico no qual a mistura reacional foi mantida sob agitação e cuja cinética foi acompanhada por intermédio de medidas simultâneas do potencial redox de um eletrodo de platina e do relativo a um eletrodo de prata/brometo de prata, ambos medidos versus um eletrodo reversível de hidrogênio. Adicionalmente, a emergência e evolução de padrões espaçotemporais auto-organizados também foram monitoradas. Nesse caso, a mistura reacional foi colocada em um reator quasibidimensional e a evolução fotografada.

\section{PARTE EXPERIMENTAL}

Utilizou-se um reator de vidro $50 \mathrm{~mL}$ de volume e tampa de teflon. Agitação foi realizada com um agitador magnético convencional. Para o monitoramento do potencial redox global da reação foi utilizado um eletrodo de platina medido versus um eletrodo padrão de hidrogênio com concentração de ácido idêntica à solução de trabalho ("Same solution hydrogen electrode, SSHE"). Adicionalmente, em alguns experimentos utilizou-se simultaneamente ao eletrodo de platina um eletrodo de prata/brometo de prata também medido versus SSHE. Em ambos os casos e os dados foram adquiridos de multímetros digitais (ET-2210 e ET-2231, da Minipa) interfaceados com microcomputadores pessoais via porta RS232-C, ou com um sistema de aquisição de dados da DATAQ (modelo DI710-UL). O eletrodo de $\mathrm{Ag} / \mathrm{AgBr}$ foi preparado segundo o procedimento adaptado da sugestão original de Woodson e Liebhfsky ${ }^{28}$. anodização galvanostática $\left(5-10 \mathrm{mAcm}^{-2}\right)$ de um eletrodo de prata em solução aquosa a $1 \mathrm{M}$ de $\mathrm{HBr}$ por alguns minutos. A solução de ferroína, um complexo composto de sulfato de ferro $\left(\mathrm{FeSO}_{4}\right.$, Vetec) e 1,10-fenantrolina (Riedel-de Haën), foi preparada momentos antes de cada experimento, na proporção estequiométrica adequada. Foram feitas três soluções, solução I: $\mathrm{NaH}_{2} \mathrm{PO}_{2}$ (Vetec), $\mathrm{MnSO}_{4}$ (Mallinckodt), $\mathrm{H}_{2} \mathrm{SO}_{4}$ (Synth) e acetona (Qhemie); solução II: $\mathrm{NaBrO}_{3}(\mathrm{Vetec})$ e solução III: ferroína, todas com água de alta pureza (sistema Milli-Q, Millipore). Inicialmente, as soluções I e II foram misturadas e, após a estabilização do sistema, adicionou-se a solução III à mistura, de forma a totalizar $50 \mathrm{~mL}$.

As investigações espaço-temporais com o sistema estagnado foram realizadas em um reator circular (c.a. $146 \mathrm{~mm}$ de diâmetro) coberto com uma placa de vidro. Após o início das oscilações no reator volumétrico a mistura foi colocada no reator quasibidimensional, formando uma camada de solução de aproximadamente 2-3 mm, e mantida sob iluminação ambiente em uma capela. A evolução espaço-temporal foi registrada com uma câmera fotográfica Casio (modelo Exilim EX-Z30). Todos os experimentos foram realizados a $23 \pm 1{ }^{\circ} \mathrm{C}$. 


\section{RESULTADOS E DISCUSSÃO}

\section{Sistema homogêneo}

Experimentos com o sistema $\mathrm{BrO}_{3}^{-} / \mathrm{H}_{2} \mathrm{PO}_{2}{ }^{-} /$acetona/Mn(II)ferroína foram realizados inicialmente com o monitoramento do potencial redox de um eletrodo de platina, medido versus um eletrodo reversível de hidrogênio. Uma série temporal típica obtida com o sistema resultante da mistura das soluções I, II e III é mostrada na Figura 1. Nessas condições, após um curto período de indução, oscilações relativamente lentas (período entre 12 e 15 min) são observadas. Após alguns ciclos a amplitude começa a cair, até um certo valor crítico a partir do qual as oscilações cessam. No ponto assinalado como A na Figura 1a, uma alíquota com 0,25 mmol de bromato é adicionada à mistura reacional. Após um novo período de indução, oscilações caracterizadas por uma maior amplitude, menor valor médio de potencial e menor período são observadas. Uma nova adição de $0,75 \mathrm{mmol}$ de íons bromato induz o aparecimento de oscilações de maior amplitude e freqüência comparável às observadas no regime imediatamente anterior. Nessas condições, o sistema oscila por pouco mais de $5 \mathrm{~h}$ e uma considerável queda do valor médio no potencial redox é observada a partir da segunda metade do período oscilatório. Transição subseqüente a um diferente estado dinâmico é observada após esse período. Nesse caso, as oscilações são observadas em uma região de potencial consideravelmente menor que as características das transições correspondentes aos pares redox $\mathrm{Mn}(\mathrm{II}) / \mathrm{Mn}$ (III) e Fe(phen) ${ }_{3}{ }_{3}+/ \mathrm{Fe}($ phen $){ }_{3}{ }^{3+}$, e correspondem a uma leve transição entre incolor (baixos valores de $\mathrm{E}_{\mathrm{Pt}}$ ) a vermelho claro (altos valores de $\mathrm{E}_{\mathrm{Pt}}$ ). Este regime é consideravelmente robusto e também ocorre em condições iniciais relativamente diferentes das mostradas na Figura 1a. Oscilações estáveis nesse regime foram observadas por até $3,5 \mathrm{~h}$. A alternativa mais plausível como responsável pelo fenômeno observado é a de que este regime oscilatório nasce a partir da morte do primeiro, ou seja, durante a exaustão do primeiro processo, são formadas espécies que tomam parte na dinâmica de outro oscilador. A explicação sugerida encontra respaldo em outros experimentos publicados recentemente ${ }^{29}$, no entanto, com as informações disponíveis seria imprudente especular sobre a natureza química das espécies envolvidas na transição mostrada aqui. Uma hipótese com base na existên-

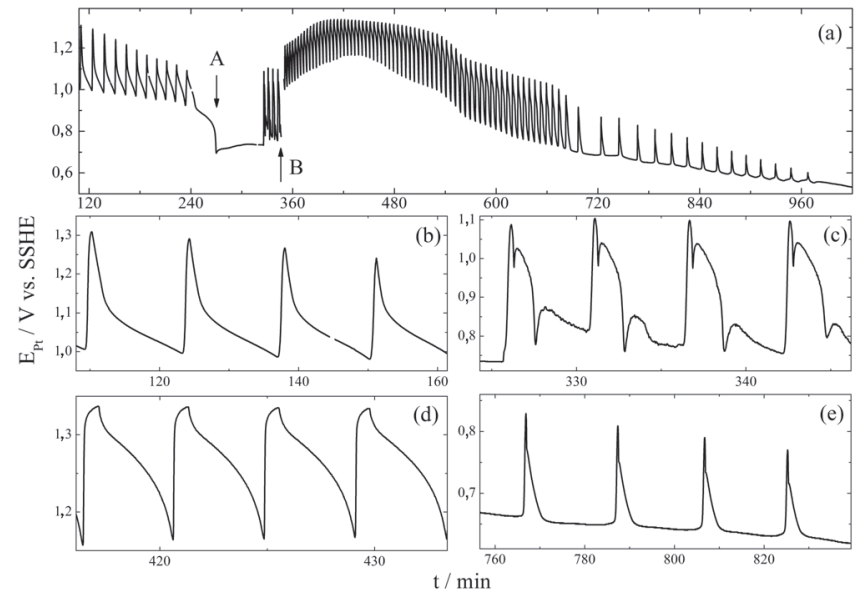

Figura 1. Evolução temporal do potencial misto medido por um eletrodo de platina com relação ao SSHE. Composição inicial: $\left[\mathrm{BrO}_{3}^{-}\right]=32 \mathrm{mM}$; $\left[\mathrm{H}_{2} \mathrm{PO}_{2}^{-}\right]$ $=110 \mathrm{mM} ;[\mathrm{Mn}(\mathrm{II})]=3,1 \mathrm{mM} ;\left[\mathrm{H}_{2} \mathrm{SO}_{4}\right]=1,33 \mathrm{M} ;$ [acetona $]=320 \mathrm{mM}$; [ferroína] = 0,33 mM. Em A foi adicionado 0,5 $\mathrm{mL}$ e em $\boldsymbol{B}$ mais 1,5 $\mathrm{mL}$ de uma solução contendo 0,5 $\mathrm{M}$ de íons bromato. Detalhes das oscilações mostradas em (a) são ilustrados nas partes $(b)-(e)$ cia de bi-ritmicidade, i.e. a coexistência entre dois ciclos-limite estáveis numa mesma região de parâmetros, pode ser excluída na explicação dessa transição, tendo em vista que o sistema é operado em batelada.

Apesar da dificuldade em se obter informações mecanísticas relevantes em experimentos realizados nas condições dos ilustrados nesta figura, i.e. com o sistema operado em batelada e com adição diferencial de um dos reagentes, é importante enfatizar a riqueza dinâmica ilustrada pela presença de variados regimes oscilatórios, como dado em detalhes nas partes (b)-(e) da Figura 1. Tal comportamento é consideravelmente mais rico que o apresentado no trabalho original ${ }^{17}$. De fato, todas as oscilações apresentadas por Orban et al. ${ }^{17}$, predominantemente utilizando $\mathrm{Ru}(\text { bpy) })_{3} \mathrm{SO}_{4}$ como segundo catalisador em lugar da ferroína, se assemelham morfologicamente às séries temporais mostradas nas Figuras $1 \mathrm{~b}$ e d. Como ilustrado nas Figuras 1 b-e, além das diferenças de amplitude e valor absoluto de potencial, as diferenças de formato e período das oscilações são bastante consideráveis. Além das oscilações mais lentas apresentadas em (e) e já discutidas, transições bastante distintas entre altos e baixos valores do potencial redox são observadas em (b) e (d). Particularmente interessante é a dinâmica presente após o tempo de indução observada após a adição de íons bromato em A e ilustrada em (c). Neste caso, as oscilações são caracterizadas por duas modulações no potencial redox, sendo uma na região de altos potenciais e a outra na de baixos. Certamente, experimentos adicionais nesta região de parâmetros seriam necessários para melhor caracterizar a aparente relaxação das modulações observada com o decorrer do tempo. Um aspecto interessante a ser levado em conta é que além das diferentes transições observadas, oscilações complexas, como a mostrada na Figura 1c, são bastante sensíveis à variação de parâmetros, além de servirem como impressão digital da dinâmica, de forma a atestar a correspondência entre resultados experimentais e simulações numéricas.

Um exemplo da influência da concentração de ácido sulfúrico é mostrado na Figura 2. O primeiro aspecto a ser notado é o aparecimento de oscilações de baixa amplitude em torno de altos valores do potencial redox durante o período de indução que precede as oscilações de grande amplitude. Tais oscilações são observadas na ausência de ferroína (adicionada apenas em $\mathrm{t}=7 \mathrm{~min}$ ) e foram mencionadas previamente ${ }^{17}$, mas encontradas aqui apenas em altas concentrações de ácido sulfúrico. Além da baixa frequiência das oscilações, as oscilações com o sistema completo, i.e. na presença do segundo catalisador, foram relativamente instáveis. Oscilações com vida curta e amplitude similar foram ainda observadas após a adição de íons bromato após a exaustão das oscilações mostradas na Figura 2.

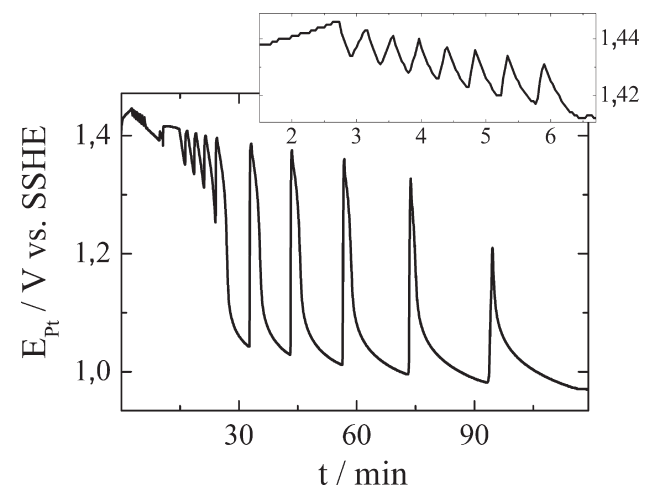

Figura 2. Evolução temporal do potencial misto medido por um eletrodo de platina com relação ao SSHE. Composição inicial: $\left[\mathrm{BrO}_{3}^{-}\right]=32 \mathrm{mM}$; $\left[\mathrm{H}_{2} \mathrm{PO}_{2}^{-}\right]$ = $110 \mathrm{mM} ;[\mathrm{Mn}(\mathrm{II})]=3,1 \mathrm{mM} ;\left[\mathrm{H}_{2} \mathrm{SO}_{4}\right]=2,5 \mathrm{M} ;$ [acetona] $=320 \mathrm{mM}$; [ferroína] $=0,33 \mathrm{mM}$ 
Apesar de adequada sob o ponto de vista qualitativo e global, a medida do potencial redox pouco informa sobre os detalhes das variações relativas das diferentes espécies presentes no meio reacional. Na tentativa de melhor inferir sobre este aspecto, adicionalmente ao monitoramento do potencial redox de um eletrodo de platina, como mostrado até aqui, medidas simultâneas do potencial de um eletrodo de $\mathrm{Ag} / \mathrm{AgBr}$ foram realizadas em alguns experimentos. Dessa forma, enquanto o eletrodo de Pt mede o potencial redox global do sistema e reflete a influência de diferentes processos, o eletrodo de $\mathrm{Ag} / \mathrm{AgBr}$, também monitorado contra o SSHE, funcionaria idealmente como eletrodo seletivo à variação de concentração de íons brometo, vide infra.

Na Figura 3 são mostrados experimentos efetuados com monitoramento simultâneo do potencial do eletrodo de platina, $\mathrm{E}_{\mathrm{Pt}}$, e de $\mathrm{Ag} / \mathrm{AgBr}, \mathrm{E}_{\mathrm{AgBr}}$. $\mathrm{O}$ sistema foi preparado com condições iniciais idênticas às utilizadas nos experimentos cujos resultados foram mostrados anteriormente na Figura 1. Na Figura 3 são explorados os aspectos da dinâmica nos instantes iniciais, i.e. $\mathrm{t}<4,5 \mathrm{~h}$, e sem a adição de íons bromato, como dado na Figura 1. Grosso modo, a exaustão do sistema ou fim das oscilações corresponde ao decréscimo dos valores médios em torno dos quais as oscilações são observadas nos dois eletrodos; obviamente, o decréscimo do $\mathrm{E}_{\mathrm{Ag} / \mathrm{AgBr}}$ corresponde a um aumento da concentração de íons brometo, de forma que o fim das oscilações corresponde ao aumento da [Br-] acima de um certo valor crítico, como amplamente conhecido nos osciladores dessa classe ${ }^{30}$.

Nas três janelas de tempo mostradas na Figura 3, os potenciais dos dois eletrodos oscilam praticamente em fase. Diferenças sutis na fase dos dois potenciais são observadas predominantemente durante a relaxação lenta para baixos valores observada no potencial redox e, mais importante, tornam-se mais acentuadas com o tempo. Equivalentemente, com base nos resultados mostrados na Figura 3, pode-se afirmar que a exaustão do sistema corresponde ao aumento da diferença de fase entre os potenciais medidos com os dois eletrodos.

Experimentos com menor concentração inicial de acetona, e também de ferroína, são mostrados na Figura 4 em termos dos potenciais $\mathrm{E}_{\mathrm{Pt}}$ e $\mathrm{E}_{\mathrm{AgBr}}$. Oscilações do tipo relaxação, de grande amplitude e baixa frequiência caracterizam a dinâmica do potencial redox. Inspeção do potencial do eletrodo de $\mathrm{Ag} / \mathrm{AgBr}$, Figura $4 \mathrm{~b}$, revela oscilações de amplitude em torno de $100 \mathrm{mV}$, consideravelmente maior que as mostradas na Figura 3. O aumento do potencial redox ocorre concomitantemente ao aumento agudo, "spikelike", do $\mathrm{E}_{\mathrm{Ag} / \mathrm{AgBr}}$. Contrastando ao mostrado na Figura 3, entretanto, os sinais medidos pelos dois eletrodos oscilam fora de fase praticamente durante todo o período de oscilação. Não obstante a diferença adicional na concentração inicial de ferroína, a diferença entre o comportamento das fases relativas nos sinais dos dois eletrodos observada a partir da comparação entre os resultados mostrados nas Figuras 3 e 4 é atribuída à menor concentração inicial de acetona utilizada nos experimentos representados na Figura 4. Argumentos para essa conjectura serão elaborados mais detalhadamente adiante.

Os autores da ref. 17 apresentaram um esquema contendo sete etapas como responsável pelas oscilações no sistema contendo bromato, hipofosfito, acetona, manganês e o complexo rutêniobipiridil. Diferentemente de propostas mecanísticas satisfatoriamente consolidadas, como por exemplo a do modelo $\mathrm{FKN}^{18}$, o esqueleto apresentado representa um esquema bem geral. Consoante a proposta sugerida ${ }^{17}$, as seguintes reações estariam envolvidas nas oscilações do sistema $\mathrm{BrO}_{3}^{-} / \mathrm{H}_{2} \mathrm{PO}_{2}^{-} /$acetona/ $/ \mathrm{Mn}$ (II)- $\mathrm{Ru}(\mathrm{bpy})_{3} \mathrm{SO}_{4}$ : $\mathrm{BrO}_{3}^{-}+4 \mathrm{Mn}(\mathrm{II})+4 \mathrm{H}^{+} \rightarrow \mathrm{HOBr}+4 \mathrm{Mn}(\mathrm{III})+2 \mathrm{H}_{2} \mathrm{O}$ $2 \mathrm{Mn}(\mathrm{III}) \rightarrow \mathrm{Mn}(\mathrm{II})+\mathrm{Mn}(\mathrm{IV})$
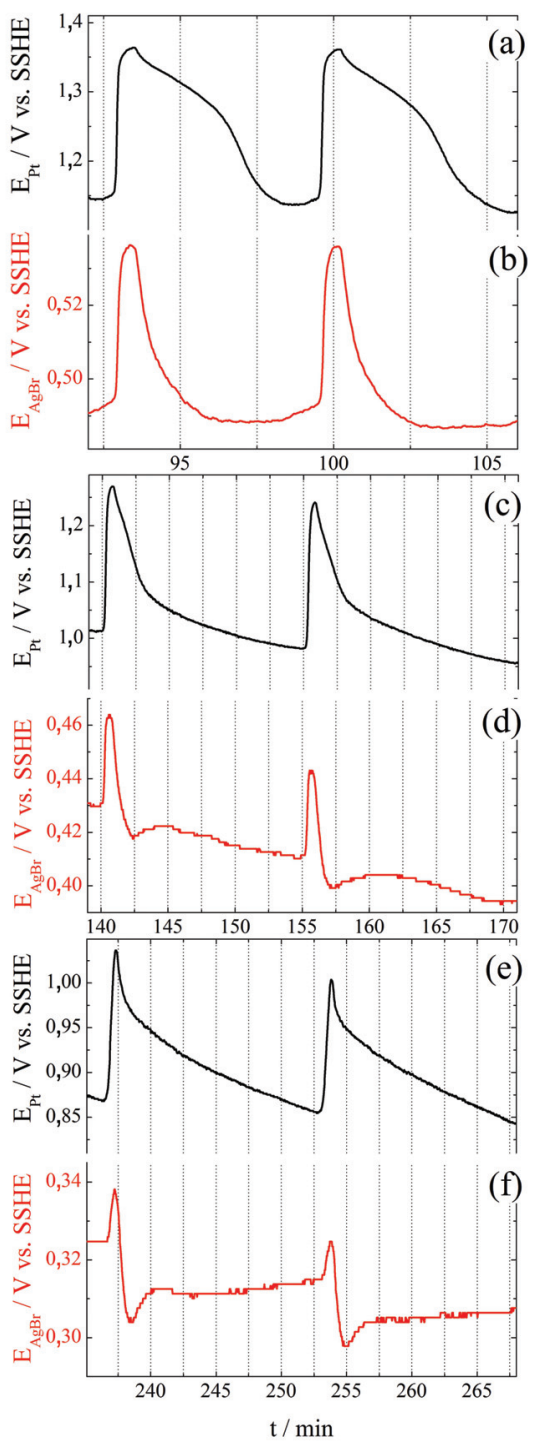

Figura 3. Evolução temporal em termos do potencial misto medido por um eletrodo de platina, $E_{P t}((a),(c)$ e (e)), e do potencial redox de um eletrodo de prata/brometo de prata, $E_{A g B r}((b),(d)$ e $(f))$, ambos em relação ao SSHE. Composição inicial: $\left[\mathrm{BrO}_{3}^{-}\right]=32 \mathrm{mM} ;\left[\mathrm{H}_{2} \mathrm{PO}_{2}^{-}\right]=110 \mathrm{mM} ;[\mathrm{Mn}(\mathrm{II})]=3,1$ $\mathrm{mM} ;\left[\mathrm{H}_{2} \mathrm{SO}_{4}\right]=1,33 \mathrm{M} ;$ [acetona $]=320 \mathrm{mM} ;[$ ferroína $]=0,33 \mathrm{mM}$

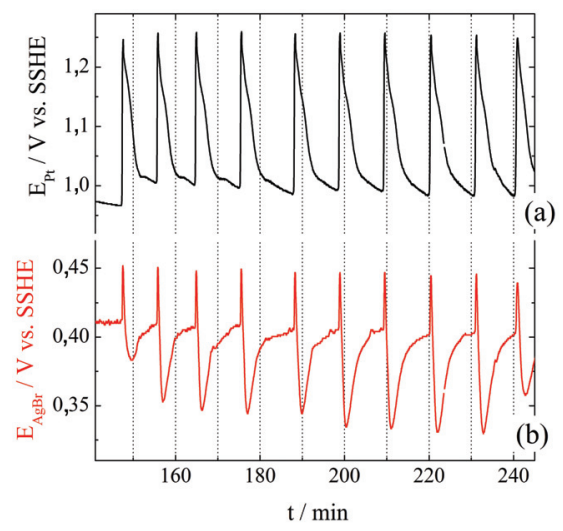

Figura 4. Evolução temporal dos potenciais $(a) E_{P_{t}}$ e (b) $E_{A_{g B} \text {, }}$, ambos medidos com relação ao SSHE. Composição inicial: $\left[\mathrm{BrO}_{3}^{-}\right]=32 \mathrm{mM} ;\left[\mathrm{H}_{2} \mathrm{PO}_{2}^{-}\right]=$ $110 \mathrm{mM} ;[\mathrm{Mn}(\mathrm{II})]=3,1 \mathrm{mM} ;\left[\mathrm{H}_{2} \mathrm{SO}_{4}\right]=1,33 \mathrm{M}$; [acetona $]=120 \mathrm{mM}$; [ferroína] $=0,1 \mathrm{mM}$ 
$\mathrm{Mn}(\mathrm{IV})+\mathrm{H}_{2} \mathrm{PO}_{2}^{-}+\mathrm{H}_{2} \mathrm{O} \rightarrow \mathrm{Mn}(\mathrm{II})+\mathrm{H}_{3} \mathrm{PO}_{3}+\mathrm{H}^{+}$

$\mathrm{HOBr}+\mathrm{Br}^{-}+\mathrm{H}+\rightarrow \mathrm{Br}_{2}+\mathrm{H}_{2} \mathrm{O}$

$\mathrm{Br}_{2}+$ Acetona $\rightarrow \mathrm{Br}-\mathrm{Ac}+\mathrm{Br}^{-}+\mathrm{H}^{+}$

(M5)

$\mathrm{Mn}(\mathrm{III})+\mathrm{Ru}(\mathrm{II}) \rightarrow \mathrm{MN}(\mathrm{II})+\mathrm{Ru}(\mathrm{III})$

(M6)

$2 \mathrm{Ru}(\mathrm{III})+2 \mathrm{Br}^{-} \rightarrow 2 \mathrm{Ru}(\mathrm{II})+\mathrm{Br}_{2}$

Um dos principais pontos a ser discutido com relação ao mecanismo proposto diz respeito ao papel da substituição do catalisador, especificamente a utilização da ferroína no presente caso. Segundo o mecanismo sugerido, além da etapa (M4) a seguinte etapa seria responsável pelo consumo de íons brometo em um sistema contendo ferroína como segundo catalisador:

$2 \mathrm{Fe}(\text { phen })_{3}{ }^{3+}+2 \mathrm{Br}^{-} \rightarrow 2 \mathrm{Fe}(\text { phen })_{3}{ }^{2+}+\mathrm{Br}_{2}$

Considerações termodinâmicas devem ser levadas em conta, tendo em vista que a etapa de redução do bromo está associada a um potencial padrão, $\mathrm{E}^{\mathrm{o}}$, de $1,09 \mathrm{~V}$,

$\mathrm{Br}_{2}+2 \mathrm{e}^{-} \rightarrow 2 \mathrm{Br}^{-}$

de forma que a etapa (M7) se torna possível pois a redução do complexo de rutênio,

$\mathrm{Ru}(\mathrm{bpy})_{3}^{3+}+\mathrm{e}^{-} \rightarrow \mathrm{Ru}(\mathrm{bpy})_{3}^{2+}$

possui potencial padrão de 1,21 V. Por outro lado, a redução da ferroína apresenta potencial padrão de $1,06 \mathrm{~V}$,

$2 \mathrm{Fe}(\text { phen })_{3}^{3+}+\mathrm{e}^{-} \rightarrow 2 \mathrm{Fe}(\text { phen })_{3}^{2+}$

e, conseqüentemente, a reação espontânea seria a produção de brometo,

$2 \mathrm{Fe}(\text { phen })_{3}{ }^{2+}+\mathrm{Br}_{2} \rightarrow 2 \mathrm{Fe}(\text { phen })_{3}^{3+}+2 \mathrm{Br}$

contrastando com seu consumo dado pela reação (1). Em termos cinéticos, Kéki et al. ${ }^{24}$ estudaram algumas reações entre ferroína/ ferriína e espécies como brometo, bromato e ácido bromoso. Uma das principais observações feita pelos autores consiste na constatação experimental de que, aparentemente em contraste ao sistema catalisado por íons cério, as espécies ferroína e ferriína apresentam acentuada reatividade frente a diferentes espécies presentes no meio reacional e não apenas ao radical $\mathrm{BrO}_{2}$. Particularmente no tocante ao problema discutido aqui, os autores observaram velocidades de reação consideravelmente maiores para a reação (2) em comparação à (1). Wang e colaboradores ${ }^{31}$ estudaram uma variante da reação de BZ utilizando ferroína e operada em batelada. Através da conjunção entre resultados experimentais e simulações numéricas, os autores sugeriram a inclusão de algumas etapas no mecanismo, além da exclusão da reação (1) como relevante à modelagem. Adicionalmente à reação (2), as seguintes reações apresentadas pelos autores podem ser consideradas relevantes ao sistema em consideração aqui ${ }^{24,31,32}$ :

$2 \mathrm{Fe}(\text { phen })_{3}{ }^{2+}+\mathrm{BrO}_{2} \cdot+\mathrm{H}^{+} \rightarrow 2 \mathrm{Fe}($ phen $){ }_{3}^{3+}+\mathrm{HBrO}^{3+}$

$2 \mathrm{Fe}(\text { phen })_{3}^{2+}+\mathrm{BrO}_{3}{ }^{-}+3 \mathrm{H}^{+} \rightarrow 2 \mathrm{Fe}(\text { phen })_{3}{ }^{3+}+\mathrm{HBrO}_{2}+\mathrm{H}_{2} \mathrm{O}$

Orban et al. ${ }^{17}$ sugeriram a seguinte reação como a fonte de íons brometo no sistema $\mathrm{BrO}_{3}-/ \mathrm{H}_{2} \mathrm{PO}_{2}^{-} /$acetona/Mn(II)- $\mathrm{Ru}(\mathrm{bpy})_{3} \mathrm{SO}_{4}$ :

$\mathrm{BrO}_{3}^{-}+3 \mathrm{H}_{2} \mathrm{PO}_{2}^{-}+3 \mathrm{H}^{+} \rightarrow \mathrm{Br}^{-}+3 \mathrm{H}_{3} \mathrm{PO}_{3}$

Tal reação foi relatada como bastante lenta e autocatalítica com relação ao brometo, e é catalisada (e oscilatória) por íons Mn(II), de forma que nem catálise nem oscilações são observadas com outros catalisadores tradicionais como Ce(III), ferroína ou complexo de rutênio ${ }^{17}$. Portanto, diferentemente do sistema original, as reações (2) e (5) podem ser consideradas fontes de brometo no sistema $\mathrm{BrO}_{3}-/ \mathrm{H}_{2} \mathrm{PO}_{2}^{-} /$acetona/Mn(II)-ferroína investigado aqui.

No mecanismo dado pelas etapas (M1) a (M7), atribuiu-se um importante papel para a acetona no sistema como sequiestrador do bromo $\left(\mathrm{Br}_{2}\right)$ formado, que é um inibidor do sistema quando está em excesso, formando a bromo-acetona. Pela estequiometria e reatividade, uma molécula de acetona poderia consumir até três moléculas de bromo, porém a bromo-acetona formada na primeira adição é volátil e grande parte se perde para a camada de ar que há acima da solução. Portanto, a acetona é responsável pela continuidade das oscilações no sistema, pois sem ela a quantidade de bromo formado seria muito grande e inibiria o sistema de oscilar.

Como apresentado na introdução, são claras as vantagens da utilização de acetona como substrato orgânico e seqüestrador de bromo em osciladores envolvendo bromato. No entanto, implicações mecanísticas adicionais relacionadas à sua utilização têm sido relatadas. O sistema bromato/ácido oxálico/acetona/Mn(II) em ácido sulfúrico operado em batelada foi recentemente investigado por Guedes e Faria ${ }^{29}$. Observou-se neste estudo que a concentração de acetona tem um impacto capital na dinâmica, implicando basicamente em uma diminuição de amplitude das oscilações com uma diminuição da concentração de acetona. Em um trabalho subseqüente, Pereira e Faria ${ }^{33}$ discutiram o papel da acetona no sistema bromato/ácido oxálico/acetona/Ce(IV) em ácido sulfúrico operando em reator agitado de fluxo contínuo ("continuous stirred tank reactor, CSTR"). Além de remover o bromo, os autores discutiram uma eventual participação da acetona na conversão lenta de Ce(IV) em $\mathrm{Ce}(\mathrm{III})$, com oscilações regulares presentes apenas a partir de um certo valor crítico da razão [Ce(III)]/[Ce(IV)].

A faixa de concentração de acetona compreendida entre 80 e $320 \mathrm{mM}$ foi indicada como ótima para a observação de oscilações no sistema bromato/hipofosfito/acetona/dois catalisadores ${ }^{17}$. Obviamente, tais valores não devem ser considerados independentemente das concentrações relativas dos outros componentes, tendo em vista que a dinâmica oscilatória resulta do acoplamento sutil entre diferentes etapas reacionais e, portanto, diferentes constantes de velocidade. No presente caso, oscilações não foram observadas quando a concentração inicial de acetona foi $80 \mathrm{mM}$ e demais condições idênticas às mostradas na Figura 3. São discutidas a seguir algumas implicações da variação da concentração de acetona na dinâmica.

Oscilações apresentadas anteriormente na Figura 4 são mostradas em termos do potencial redox e da concentração de íons brometo na Figura 5. Nessa representação pode-se observar que o aumento do potencial redox está correlacionado com uma pequena queda na concentração de íons brometo, que por sua vez experimenta um grande aumento durante a queda inicial do valor do potencial redox. Análise similar com os resultados mostrados na Figura 3, na qual uma concentração inicial de acetona de $320 \mathrm{mM}$ foi empregada, revelaram que as oscilações na concentração de íons brometo ocorrem praticamente fora de fase com o potencial redox e que, mais importante, ocorre em torno de um valor médio significativamente menor que o mostrado na Figura 5. Dessa forma, o papel da acetona no controle das oscilações pode ser primariamente identificado pela faixa de concentração das oscilações de brometo, de forma que, quanto menor sua concentração maior é a concentração de íons brometo.

Para excluir algum eventual efeito da menor concentração inicial de ferrroína, empregada no experimento mostrado na Figura 5, nos altos valores da concentração de íons brometo, resultados obtidos em condições idênticas às mostradas na Figura 3, mas com 


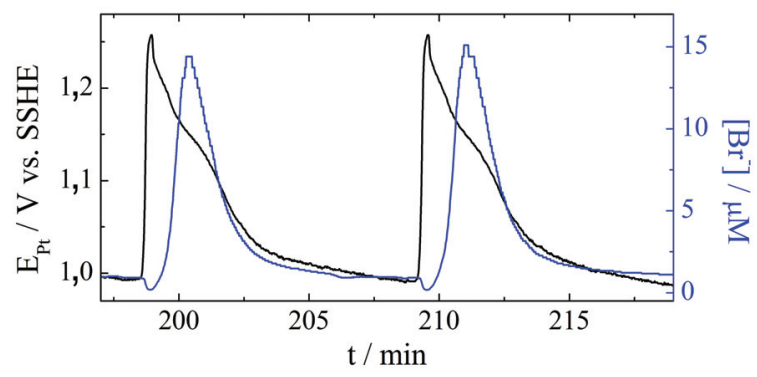

Figura 5. Evolução temporal do $E_{P_{t}}$ (em preto), e da $\left[\mathrm{Br}^{-}\right]$(em azul). Composição: $\left[\mathrm{BrO}_{3}^{-}\right]=32 \mathrm{mM} ;\left[\mathrm{H}_{2} \mathrm{PO}_{2}^{-}\right]=110 \mathrm{mM} ;[\mathrm{Mn}(\mathrm{II})]=3,1 \mathrm{mM}$; $\left[\mathrm{H}_{2} \mathrm{SO}_{4}\right]=1,33 \mathrm{M} ;$ [acetona $]=120 \mathrm{mM} ;[$ ferroína $]=0,1 \mathrm{mM}$

concentração inicial de acetona de $120 \mathrm{mM}$, são ilustrados na Figura 6. Nesse caso, tendo em mente que a menor concentração de acetona implica uma menor estabilidade das oscilações, a maior concentração inicial de ferroína resulta na observação de oscilações transientes e a concentração de íons brometo aumenta rapidamente, promovendo o fim das oscilações. Os altos valores da concentração de íons brometo parecem implicar ainda na menor amplitude do potencial redox, como aparente nas Figuras 5 e 6 .

A obtenção da concentração de íons brometo a partir dos valores de potencial do eletrodo de $\mathrm{Ag} / \mathrm{AgBr}$ foi feita através da equação de Nernst $^{34}$, desprezando as, certamente presentes nas condições de realização dos experimentos $^{34,35}$, não idealidades. Adicionalmente, uma nota de precaução deve ser feita com relação à utilização dos valores de $[\mathrm{Br}$ ] mostrados. Infelizmente, na presença de algumas espécies no meio reacional, a relação entre a variação de potencial do eletrodo de $\mathrm{Ag} /$ $\mathrm{AgBr}$ e a concentração de brometo no seio da solução pode diferir da prevista pela equação de Nernst. De fato, como discutido por Noszticzius et al. ${ }^{36,37}$, a presença de ácidos hipobromoso $(\mathrm{HOBr})$, bromoso $\left(\mathrm{HBrO}_{2}\right)$ e brômico $\left(\mathrm{HBrO}_{3}\right)$ no meio reacional pode provocar a corrosão do eletrodo de $\mathrm{Ag} / \mathrm{AgBr}$, removendo íons brometo da superfície. Como resultado tem-se geralmente uma situação na qual o potencial do eletrodo se torna independente da concentração de brometo no seio da solução, passando a depender da concentração do agente corrosivo. Em geral, esse problema se torna particularmente importante quando a concentração de brometo é menor que seu o limite de solubilidade $(1,54 \mu \mathrm{M})$. Para concentrações acima desse valor, os resultados obtidos com o eletrodo de $\mathrm{Ag} / \mathrm{AgBr}$ são relativamente confiáveis e, pelo menos de forma qualitativa, servem para inferir sobre as variações na concentração de íons brometo. Quanto ao íon $\mathrm{Ag}^{+}$, ainda pouco se conhece da interferência deste íon em solução ${ }^{38} \mathrm{e}$, por isso, pode ser necessário fazer os experimentos com o monitoramento do eletrodo de prata através de uma ponte salina. Apesar de importante como técnica complementar, a utilização de eletrodos seletivos não parece adequada a situações que requerem maior precisão como no caso de determinações cinéticas. Para esse fim, muito mais apropriado seria a utilização de métodos espectrofotométricos ${ }^{14}$. Particularmente nos experimentos de interesse aqui, informações importantes seriam obtidas através do acompanhamento, por exemplo, das concentrações de Mn(IV) e ferroína determinadas a 380 e $490 \mathrm{~nm}$, respectivamente ${ }^{39,40}$. Dado o exposto, a análise dos dados da concentração de íons brometo deve ser feita de forma apenas semi-quantitativa e é justificada pela faixa de concentração obtida (Figuras 5 e 6).

\section{Sistema espacialmente estendido}

Além dos experimentos conduzidos no reator volumétrico mantido sob agitação e discutidos até aqui, experimentos com o sistema estagnado na configuração de camada fina de solução também foram reali-

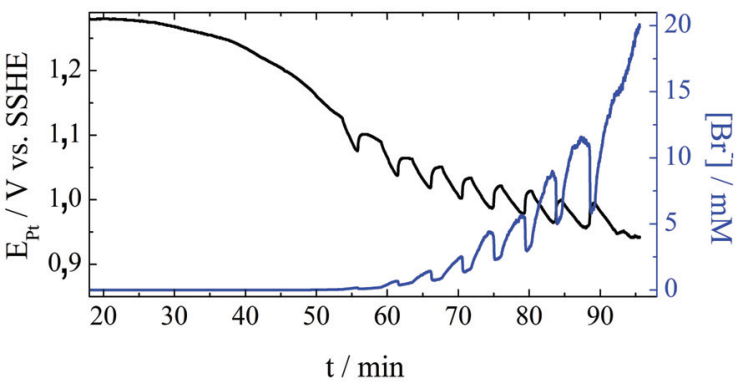

Figura 6. Evolução temporal do $E_{P_{t}}$ (em preto), e da [Br-] (em azul). Composição: $\left[\mathrm{BrO}_{3}^{-}\right]=32 \mathrm{mM} ;\left[\mathrm{H}_{2} \mathrm{PO}_{2}^{-}\right]=110 \mathrm{mM} ;[\mathrm{Mn}(\mathrm{II})]=3,1 \mathrm{mM}$; $\left[\mathrm{H}_{2} \mathrm{SO}_{4}\right]=1,33 \mathrm{M} ;$ [acetona $]=120 \mathrm{mM} ;[$ ferroína $]=0,33 \mathrm{mM}$

zados. Nesses experimentos, após o início das oscilações no reator volumétrico, a mistura reacional foi colocada em um reator quasibidimensional encerrando uma espessura aproximada entre $2-3 \mathrm{~mm}$. Em linhas gerais, os padrões obtidos foram de boa qualidade, apresentando frentes reacionais relativamente nítidas e com um bom contraste. Assim como relatado previamente ${ }^{17}$, não foi observada a formação de bolhas e precipitados durante esses experimentos.

A sequiência de imagens mostrada na Figura 7 indica a evolução de padrões em forma de alvo ("target patterns") durante aproximadamente um período de oscilação (16 min). Padrões em forma de alvo emergem espontaneamente pouco após a mistura reacional ser espalhada na placa. $\mathrm{O}$ comprimento de onda de tais estruturas foi estimado como sendo da ordem de 2 a $3 \mathrm{~cm}$, enquanto as frentes reacionais se propagam com velocidade constante de aproximadamente $0,2 \mathrm{~cm} \mathrm{~min}^{-1}$.

Quebra da simetria anular dos alvos pode ser feita através de perturbação mecânica, gentilmente com um fio de platina como no presente caso, por exemplo. Como resultado tem-se a formação típica de ondas espiraladas nos estágios iniciais de evolução dos alvos seguida pela sucessiva quebra dos segmentos da frente reacional e distorção da propagação com a co-existência de diferentes comprimentos de onda, como mostrado na sequiência dada na Figura 8. Considerando a grande simetria anular de propagação presente no caso anterior, Figura 7, não se pode afirmar que a observação de diferentes velocidades de propagação vistas na sequiência mostrada na Figura 8 esteja ligada a qualquer anisotropia presente no meio.

A observação de aspectos adicionais da dinâmica espaço-temporal se torna mais transparente a partir da utilização de representação bi-dimensional da evolução espacial em função do tempo. Tal representação foi construída através do acompanhamento da evolução espacial em uma região selecionada por uma fatia retangular quasi-unidimensional (de largura $\mathrm{x}=0,1 \mathrm{~cm}$ e altura $\mathrm{y}=14,6 \mathrm{~cm}$ ) posicionado no centro do reator, nas proximidades do centro da espiral superior mostrada na Figura 7a. Os resultados são mostrados na Figura 9 e incluem a seqüência das estruturas mostradas nas Figuras 7 e 8 . Os resultados mostrados foram obtidos a partir do início das oscilações e demonstram claramente a co-existência entre a região nas quais as ondas químicas são observadas (metade superior) e a região na qual oscilações praticamente homogêneas prevalecem (metade inferior, y < c.a. $6 \mathrm{~cm}$ ). A análise das oscilações nessa região indica claramente um período aproximado de 16 min.

Como já indicado pela contínua expansão da região na qual quebra de simetria ocorre, com o passar do tempo, as ondas reacionais invadem e dominam todo o meio, seja através dos alvos ou das espirais. Adicionalmente à expansão do domínio não-homogêneo, observa-se ainda uma tendência à formação de modulações espaciais acentuadas, em particular na região aproximada $0<\mathrm{y}<6$ $\mathrm{cm}$ e $1,5<\mathrm{t}<2 \mathrm{~h}$. Na metade superior da evolução espaço-tempo- 


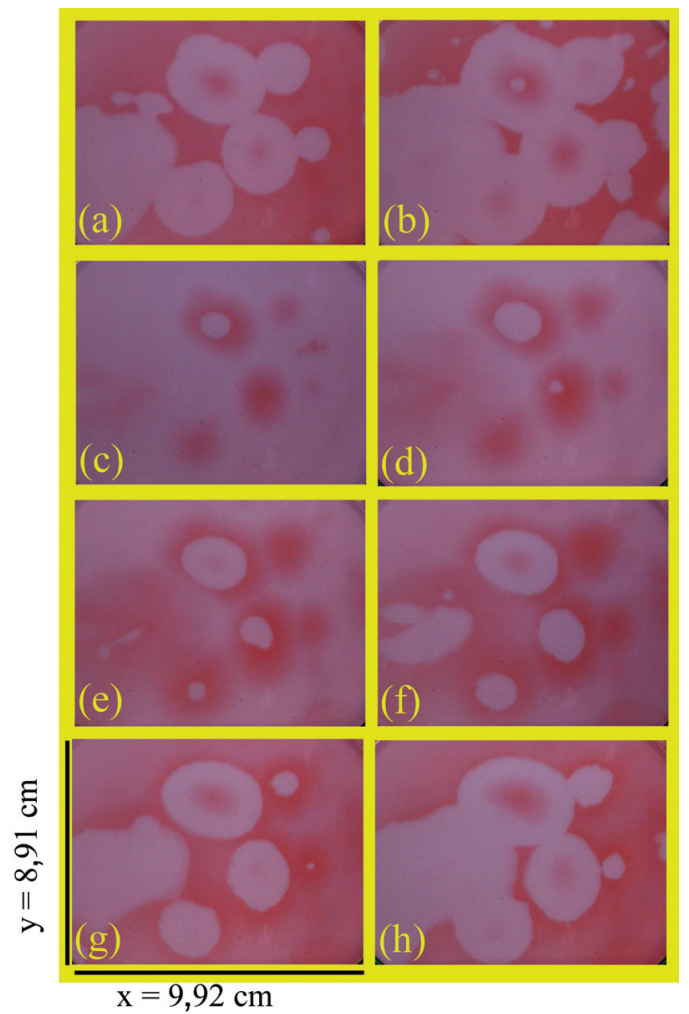

Figura 7. Evolução espaço-temporal de padrões em forma de alvo em regime oscilatório. Composição da mistura reacional: $\left[\mathrm{BrO}_{3}^{-}\right]=32 \mathrm{mM}$; $\left[\mathrm{H}_{2} \mathrm{PO}_{2}^{-}\right]$ $=113 \mathrm{mM} ;[\mathrm{Mn}(\mathrm{II})]=3,1 \mathrm{mM} ;\left[\mathrm{H}_{2} \mathrm{SO}_{4}\right]=1,33 \mathrm{M} ;[$ acetona $]=320 \mathrm{mM}$; [ferroína] = 0,40 mM. Intervalo de 2 min entre as fotos. A escala entre os eixos $x$ e y não é linear pois as imagens foram registradas com a câmera posicionada a $26^{\circ}$ em relação ao eixo normal à superfície

ral mostrada na Figura 9, é detalhada a evolução dos alvos, Figura 7, assim como das estruturas obtidas após a perturbação em torno de $\mathrm{t}=70 \mathrm{~min}$, Figura 8. Após uma pequena variação da posição do marca-passo para valores maiores de y no início da evolução, os alvos se tornam estáveis até o momento da perturbação e apenas breve desvios do centro são observados.

Além dos padrões concêntricos em forma de alvo e das espirais mais comumente observados e ilustrados até aqui, observou-se ainda a formação de estruturas estriadas com comprimento de onda bastante regular durante a propagação de frentes reacionais das bordas para o centro da placa. No entanto, ao contrário das estruturas mostradas nas Figuras 7 e 8, a espessura da camada de solução foi ligeiramente mais espessa e não se pôde excluir a possibilidade de acoplamento entre o efeito convectivo perpendicular à propagação da estrutura e a instabilidade química inerente a sistemas de reaçãodifusão. Entretanto, antes de qualquer explicação mais fundamentada experimentos adicionais, em andamento, são necessários.

\section{CONCLUSÕES}

Foram estudados neste trabalho alguns aspectos da dinâmica espaço-temporal do sistema bromato/hipofosfito/acetona/dois catalisadores. Diferentemente do relatado inicialmente por Orban et $a l .{ }^{17}$, as investigações apresentadas aqui foram centradas no sistema composto por manganês e ferroína como catalisadores. Uma rica dinâmica foi observada, incluindo diferentes modos e a presença de oscilações complexas, ausentes no trabalho anterior com o sistema utilizando um complexo de rutênio como segundo catalisador. Nesse sentido, oscilações como as ilustradas na Figura 1c são bastante sen-

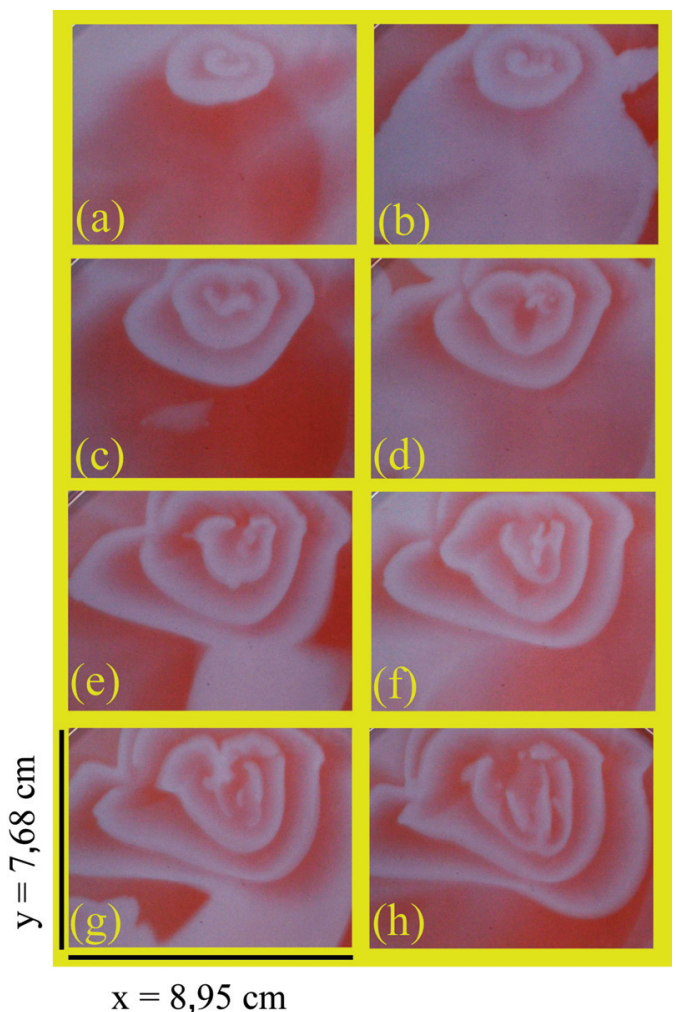

Figura 8. Evolução espaço-temporal de padrões em forma de alvo em regime oscilatório. Composição da mistura reacional: $\left[\mathrm{BrO}_{3}^{-}\right]=32 \mathrm{mM} ;\left[\mathrm{H}_{2} \mathrm{PO}_{2}^{-}\right]$ $=113 \mathrm{mM} ;[\mathrm{Mn}(\mathrm{II})]=3,1 \mathrm{mM} ;\left[\mathrm{H}_{2} \mathrm{SO}_{4}\right]=1,33 \mathrm{M} ;$ [acetona $]=320 \mathrm{mM}$; [ferroína] =0,40 $\mathrm{mM}$. Intervalo de 10 min entre as fotos. A escala entre os eixos x e y não é linear pois as imagens foram registradas com a câmera posicionada a $26^{\circ}$ em relação ao eixo normal à superfície

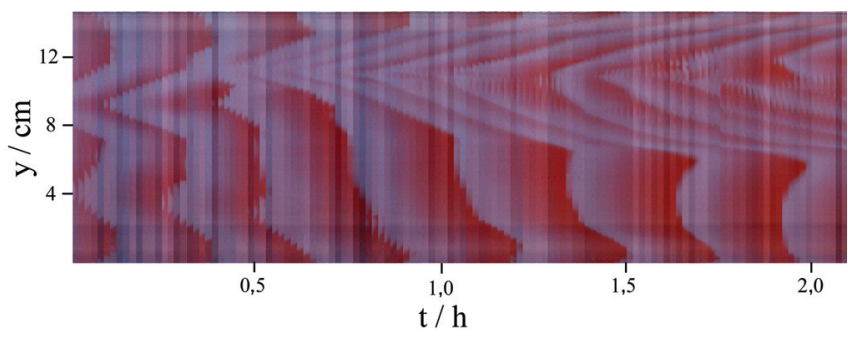

Figura 9. Evolução espaço-temporal de padrões em forma de alvo em regime oscilatório. Composição da mistura reacional: $\left[\mathrm{BrO}_{3}^{-}\right]=32 \mathrm{mM} ;\left[\mathrm{H}_{2} \mathrm{PO}_{2}^{-}\right]$ = $113 \mathrm{mM} ;[\mathrm{Mn}(\mathrm{II})]=3,1 \mathrm{mM} ;\left[\mathrm{H}_{2} \mathrm{SO}_{4}\right]=1,33 \mathrm{M} ;$ [acetona $]=320 \mathrm{mM}$; [ferroína] $=0,40 \mathrm{mM}$. Intervalo de $10 \mathrm{~min}$ entre as fotos. A escala entre os eixos $x$ e y não é linear pois as imagens foram registradas com a câmera posicionada a $26^{\circ}$ em relação ao eixo normal à superfície

síveis à variação de parâmetros e servem como impressão digital da dinâmica associada, de forma a atestar a correspondência entre resultados experimentais e simulações numéricas. Em contraste à redução do catalisador secundário gerando bromo, a oxidação da ferroína à ferriína atua como fonte adicional de íons brometo. Finalmente, foi observado que uma baixa concentração inicial de acetona implica em uma baixa estabilidade das oscilações e é refletida em uma alta concentração de brometo. O final das oscilações ocorre quando a concentração de brometo ultrapassa um certo valor crítico.

Foram realizados ainda experimentos em uma configuração quasi-bidimensional, em regime oscilatório. Os padrões espaço-temporais observados apresentaram bom contraste, ausência de produ- 
tos gasosos e sólidos, além de longo tempo de oscilação. Estruturas em forma de alvo com comprimento de onda entre 2 a $3 \mathrm{~cm}$ com frentes reacionais com velocidade constante de aproximadamente $0,2 \mathrm{~cm} \mathrm{~min}^{-1}$ emergiram espontaneamente quando a mistura reacional foi encerrada no reator. Perturbação dos alvos geraram a formação de espirais instáveis. Em alguns casos, oscilações homogêneas localizadas co-existiram com alvos e espirais.

Considerando a simplicidade de preparação e manipulação, robustez, variedade dinâmica, estabilidade, ausência de formação produtos gasosos e sólidos, e longo tempo de oscilações quando operado em batelada, o sistema bromato/hipofosfito/acetona/manganês e ferroína mostrou-se bastante adequado ao estudo da dinâmica espaçotemporal em sistemas químicos oscilantes. Devido à fotossensibilidade, o sistema espacialmente estendido parece ainda bem apropriado a estudos futuros sobre o impacto do acoplamento global na emergência, seleção e evolução de estruturas auto-organizadas.

\section{AGRADECIMENTOS}

À Fundação de Amparo à Pesquisa do Estado de São Paulo (FAPESP) pelo auxílio financeiro (04/04528-0) e bolsa (G. Tokoro: 05/03369-9) concedidos, e ao CNPq.

\section{REFERENNCIAS}

1. Singh, R.; Maru, V. M.; Moharir, O. S. ; J. Nonlin. Sci. 1998, 8, 235.

2. Korn, R.W.; Biology and Philosophy 2005, 20, 137.

3. Anderson, P. W.; Science 1972, 177, 393.

4. Whitesides, G. M.; Ismagilov, R. F.; Science 1999, 284, 89.

5. Shanks, N.; Found. Chem. 2001, 3, 33.

6. Müller, S. C.; Hauser, M. J. B. Em Handbook of Biomimetics; Osaka, Y; Kai, S.; Kakazu, Y.; Kataoka, K.; Sakai, K.; Tanaka, J., eds.; NTS Books: Tokio, 2000, p. 87

7. Ertl, G.; Science 1991, 254, 1750

8. Rotermund, H. H.; Engel, W.; Kordesch, M.; Ertl, G.; Nature 1990, 343, 355.

9. Imbihl, R.; Ertl, G.; Chem. Rev. 1995, 95, 697.

10. Li, Y. J.; Oslonovitch, J.; Mazouz, N.; Plenge, F.; Krischer, K.; Ertl, G.; Science 2001, 291, 2395.
11. Varela, H.; Beta, C.; Bonnefont, A.; Krischer, K.; Phys. Rev. Lett. 2005, 94, 174104-1.

12. Varela, H.; Beta, C.; Bonnefont, A.; Krischer, K.; Phys. Chem. Chem. Phys. 2005, 7, 2429 .

13. Zhabotinsky, A. M.; Chaos 1991, 1, 379.

14. Taylor, A. F.; Prog. Reac. Kin. Mech. 2002, 27, 247.

15. Faria, R. B.; Quim. Nova 1995, 18, 281.

16. Noyes, R. M.; J. Am. Chem. Soc. 1980, 102, 4644.

17. Orban, M.; Kurin-Csorgei, K.; Zhabotinsky, A. M.; Epstein, I. R.; Faraday Discuss. 2001, 120, 11

18. Field, R. J.; Korös, E.; Noyes, R. M.; J. Am. Chem. Soc. 1972, 94, 8649

19. Kurin-Csörgei, K.; Orban, M.; Zhabotinsky, A. M.; Epstein, I. R.; J. Phys. Chem. A 1997, 101, 6827.

20. Ouyang, Q.; Tam, W. Y.; DeKepper, P.; McCormick, W. D.; Noszticzius, Z.; Swinney, H. L.; J. Phys. Chem. 1987, 91, 2181.

21. Bowers, P. G.; Caldwell, K. E.; Prender, D. F.; J. Phys. Chem. 1972, 76, 2185.

22. Salter, L. F.; Sheppard, J. G.; Int. J. Chem. Kinet. 1982, 14, 815.

23. Sridevi, V.; Ramaswamy, R.; Int. J. Chem. Kinet. 1998, 30, 201.

24. Kéki, S.; Magyar, I.; Beck, M. T.; Gaspar, V.; J. Phys. Chem. 1992, 96, 1725 .

25. Winfree, A. T.; Caudle, S.; Chen, G.; McGuirre, P.; Szilagyi, Z.; Chaos 1996, 6, 617.

26. Tóth, R.; Gaspar, V.; Belmonte, A.; O’Connell, M. C.; Taylor, A.; Scott, S. K.; Phys. Chem. Chem. Phys. 2000, 2, 413.

27. Plenge, F.; Varela, H.; Krischer, K.; Phys. Rev. Lett. 2005, 94, 198301-1.

28. Woodson, J. H.; Liebhfsky, H. A.; Anal. Chem. 1969, 41, 1894.

29. Guedes, M. C.; Faria, R. B.; J. Phys. Chem. A 1998, 102, 1973.

30. Scott, S. K.; Chemical Chaos, Clarendon Press: Oxford, 1991.

31. Wang, J.; Zhao, J.; Chen, Y.; Gao, Q.; Wang, Y.; J. Phys. Chem. A 2005, $109,1374$.

32. Szalai, I.; Kurin-Csörgei, K.; Orban, M.; Phys. Chem. Chem. Phys. 2002, 4, 1271.

33. Pereira, J. A. M.; Faria, R. B.; J. Braz. Chem. Soc. 2004, 15, 976.

34. Lewis, G. N.; Randall, M.; Thermodynamics, $2^{\text {nd }}$ ed, McGraw-Hill Boook Company: New York 1961

35. Skoog, D. A.; West, D. M.; Holler, F. J.; Fundamentals of Analytical Chemistry, $7^{\text {th }}$ ed., Saunders College Publishing: Fort Worth, 1996.

36. Noszticzius, Z.; Noszticzius, E.; Schelly, Z. A.; J. Am. Chem. Soc. 1982, $104,6194$.

37. Noszticzius, Z.; Noszticzius, E.; Schelly, Z. A.; J. Phys. Chem. 1983, 87, 510.

38. Taylor, A. F.; Prog. React. Kinet. Mech. 2002, 27, 247.

39. Horvath, O.; Strohmayer, K.; J. Photochem. Photobiol., A 1998, 116, 69.

40. Kurin-Csörgei, K.; Epstein, I. R.; Orban, M.; J. Phys. Chem. B 2004, 108, 7352 . 